\title{
Efficacy of intra-articular magnesium for postoperative analgesia in total hip arthroplasty
}

\author{
XINXIAN XU, HONG WEN, YUEZHENG HU, ZHONGTANG LIU and XIAOYUN PAN \\ Department of Osteopathy, The Second Affiliated Hospital of \\ Wenzhou Medical University, Wenzhou, Zhejiang 325000, P.R. China
}

Received September 27, 2016; Accepted November 17, 2016

DOI: $10.3892 /$ br.2017.837

\begin{abstract}
The aim of the present study was to compare the efficacy of intra-articular magnesium sulphate and a saline placebo for postoperative pain control following total hip arthroplasty (THA). Sixty patients underwent THA and were randomly allocated into two groups to receive intra-articular injections of either $10 \mathrm{ml}$ magnesium sulphate $(100 \mathrm{mg} / \mathrm{ml}$; magnesium group, $\mathrm{n}=30$ ) or $10 \mathrm{ml}$ normal saline solution (control group, $\mathrm{n}=30$ ). Postoperative analgesia was maintained by intravenous morphine injection. The outcome measurements were visual analogue score (VAS), morphine consumption and Harris hip score (HHS). The two groups were well matched. The outcome of VAS at rest was significantly lower at postoperative hours 6 and 12 in the magnesium group as compared with the control group, although the difference was insignificant preoperatively and at postoperative hours 2, 4, 24 and 48, and days 3, 7 and 14. This indicator during activity was also lower in the magnesium group at postoperative hour 24 than that of the control group, although the difference was insignificant preoperatively and at hour 48 , and days 7 and 14. The consumption of morphine (the total quantity) at 0-6, 6-12 and 0-48 $\mathrm{h}$ in the magnesium group was significantly lower than in the control group, although no significant differences were observed at 12-24 and 24-48 h between the groups. The improvements of HHS from preoperative to postoperative scores were statistically significant, however, no significant differences were identified between groups. Thus, the findings indicate that intra-articular magnesium sulphate injections provided improved pain control and reduced the need for morphine when compared with a saline placebo following THA.
\end{abstract}

\section{Introduction}

Total hip arthroplasty (THA) is an effective surgical intervention for pain relief and function improvement in elderly

Correspondence to: Dr Xiaoyun Pan, Department of Osteopathy, The Second Affiliated Hospital of Wenzhou Medical University, 109 Xueyuan West Road, Wenzhou, Zhejiang 325000, P.R. China

E-mail: 379806041@qq.com

Key words: total hip arthroplasty, intra-articular, magnesium sulphate, analgesic patients presenting with hip degeneration. However, a variable amount of pain often accompanies this procedure postoperatively $(1,2)$. Inadequate control of postoperative pain may influence early rehabilitation and result in delayed recovery and prolonged hospitalization. Consequently, various studies have been conducted to obtain an ideal analgesic strategy, which could offer satisfactory analgesia with high safety.

Traditionally, post-THA analgesics include oral and epidural analgesics, peripheral nerve blockers, and intra-articular analgesia infusions, among which local analgesic injection is a simple and effective method. At present, numerous options, including opioids, non-steroidal antiinflammatory agents and local anesthetics are available for local injection (3-5). A relatively novel approach for peripheral pain control is to use magnesium sulphate. A recent study revealed magnesium had antinociceptive effects in animal and human models of chronic pain (6). It was shown to be effective as a postoperative analgesic in orthopedic surgery $(7,8)$. The analgesic property of magnesium is associated with inhibition of the N-methyl-D-aspartate (NMDA) receptor and modulation of calcium channels (9). Furthermore, its addition to local anesthetics prolongs anesthesia duration and maximizes their effects $(10,11)$. Clinical studies demonstrated that intra- articular injection of magnesium effectively ameliorated postoperative pain in arthroscopic knee surgery when compared with a placebo $(12,13)$. However, to the best of our knowledge, its application to THA has not yet been reported.

Therefore the present study was performed to compare and analyze the analgesic efficacy and safety of an intra-articular magnesium sulfate injection with a placebo following THA. It was hypothesized that patients receiving the intra-articular magnesium sulfate injection would experience reduced postoperative pain when compared with the control group.

\section{Materials and methods}

Participants. Between October 2012 and June 2014, a total of 60 patients underwent THA in the Second Affiliated Hospital of Wenzhou Medical University (Wenzhou, China) and met the inclusion criteria, thus were included in the current study. All procedures were conducted in accordance with the ethical standards of the responsible committee on human experimentation (institutional and national) and with the Helsinki Declaration of 1964 and its later amendments. Informed consent was 
obtained from all the patients included in the study. The compliance was considered to be good, as the majority of the outcome measurements were short-term assessments.

Patients who underwent unilateral THA for hip osteoarthritis or aseptic necrosis of the femoral head were eligible for the current study. The diagnosis was predominantly determined by local radiographs, and further confirmed with the help of medical history, clinical examination, and additional imaging examinations, such as computed tomography or magnetic resonance imaging if necessary. The inclusion criteria were as follows: Patients aged $<80$ years, weighing $<80 \mathrm{~kg}$, and with an American Society of Anesthesiologists physical status class I or II (14). The exclusion criteria were as follows: i) History of bleeding diathesis or any previous thromboembolic episodes; ii) recent use of therapeutic agents that may distort the results; iii) exhibiting metabolic diseases, such as hepatic or renal dysfunction, or serious cardiac disorders; iv) exhibiting hypermagnesemia or associated diseases with the potential to develop a hypermagnesemia; v) known allergies to one of the medications included in the study.

Interventions. All patients were randomized into one of two groups using computer-generated numbers. The magnesium group $(n=30)$ received $10 \mathrm{ml} \mathrm{10 \%} \mathrm{magnesium} \mathrm{sulphate} \mathrm{and} \mathrm{the}$ control group $(n=30)$ received $10 \mathrm{ml}$ normal saline solution. All patients received celecoxib (COX-2 inhibitor; PPLLC, Dalian, China) postoperatively with a dose of $200 \mathrm{mg}$ twice a day. The patients received one dose of cefazolin $(2 \mathrm{~g}$; Reyoung Pharmaceutical Co., Shandong, China) intraoperatively, and the subsequent 4 doses of the same quantity over $48 \mathrm{~h}$ postoperatively. A daily treatment of 4,000 IU low molecular weight heparin was administrated subcutaneously for deep venous thrombosis (DVT) prophylaxis.

Prior to surgery, all patients received an intravenous bolus of $500 \mathrm{ml}$ Ringer's lactate solution (Kelun Pharmaceutical Co., Sichuan, China). General anesthesia was required for all patients and all procedures were performed by the same senior anesthetist. It was induced by total intravenous anesthesia with propofol (2 mg/kg; Jiabo Pharmaceutical Co., Guangdong, China) and remifentanil (3 $\mu \mathrm{g} / \mathrm{kg}$; Nhwa Pharmaceutical Co., Jiangsu, China), and maintained with nitrous oxide in oxygen (60\%) and isoflurane (1-2\%; Lenuo Kefeng Pharmaceutical Co., Shandong, China). The depth of anesthesia was maintained by adjusting the percentage of isoflurane. Vital signs, such as heart rate (HR), blood pressure, respiratory rate and arterial oxygen saturation were monitored continuously throughout surgery. Hypotension (systolic blood pressure $<80 \mathrm{mmHg}$ or a $30 \%$ decrease from baseline) was treated with Ringer's lactate solution, if required followed by $5 \mathrm{mg}$ intravenous epinephrine (Kingyork Pharmaceutical Co., Tianjin, China). Bradycardia (HR $<50 \mathrm{bpm}$ ) was treated with $0.2 \mathrm{mg}$ atropine (Kingyork Pharmaceutical Co.).

All surgical procedures were performed by the same senior surgeon according to standard surgical routines. Metal-on-metal total hip systems without bone cement (Smith \& Nephew, Memphis, TN, USA) were available for replacement. The posterolateral approach was adopted, and a standard silicon drain with an inner diameter of $16 \mathrm{~mm}$ was placed before closing the skin, which was clamped for $2 \mathrm{~h}$ postoperatively and subsequently released. Following closure of the capsule the test medication was administrated. Patients in the magnesium group received $10 \mathrm{ml}$ magnesium sulphate solution (10\% magnesium sulphate; $1 \mathrm{~g}$ diluted in $10 \mathrm{ml}$ normal saline) intra-articularly and those in the control group received the same quantity of normal saline solution. All cases were transferred to the post-anesthesia care unit and observed for at least $2 \mathrm{~h}$ before being returned to the ward.

Morphine injection (10 mg) was administered intravenously as an analgesic supplement once a day for $48 \mathrm{~h}$ after surgery. If pain remained intolerable, an additional $5 \mathrm{mg}$ per administration was used and the total quantity was dependent on the severity and general condition of the case. No other analgesics were used during the study period. The drain at the surgical site was removed $48 \mathrm{~h}$ postoperatively. The rehabilitation protocol was identical for every patient. On postoperative day 1 , the anteroposterior and lateral radiographic views of the affected hip were reexamined. Full weight bearing was permitted after removal of the drains. The patients were permitted to be discharged when pain was manageable with oral morphine and when they were able to walk using crutches.

Outcome assessment. Preoperative variables, including age, gender, diagnosis, body mass index (BMI) and surgical duration were evaluated for each patient. The primary outcome was pain, as assessed using the visual analogue score (VAS), which ranged from $0 \mathrm{~mm}$ (representing no pain) to $100 \mathrm{~mm}$ (representing the worst pain. VAS was determined preoperatively and at hours 2, 4, 6, 12, 24 and 48, and on days 3, 7 and 14 at rest, and postoperatively at hours 24 and 48, and days 7 and 14 during activity. The secondary outcomes included morphine consumption and Harris hip score (HHS) (15). Morphine consumption was calculated preoperatively and at hours 6,12 , 24 and 48, and HHS was documented preoperatively, and at day 7 and 14 postoperatively. Serum magnesium concentration was assessed in each patient. The blood samples $(10 \mathrm{ml})$ for calculating serum magnesium concentration were obtained preoperatively and at hours 6 and 24 postoperatively. Adverse events (AEs) were recorded within 14 postoperative days.

Statistically analysis. All data were analyzed by the research team of our department in conjunction with a medical statistician using the latest version of SPSS 19.0 (IBM SPSS, Armonk, NY, USA). Continuous data are presented as means \pm standard deviation and analyzed using paired or unpaired Student's t-test. Categorical data are presented using proportions and analyzed using the $\chi^{2}$ test with Fisher's exact test. $\mathrm{P}<0.05$ was considered to indicate a statistically significant difference.

\section{Results}

Baseline demographic characteristics and clinical data. All patients completed the study protocol. The baseline demographic characteristics and clinical data are presented in Table I. Two groups of patients were well matched, with no significant differences in age, gender, BMI, diagnosis, length of surgery, serum magnesium concentration and total drainage fluid observed between the two groups.

Outcome of VAS, consumption of morphine and HHS. The outcome of VAS at rest was significantly lower at postoperative 
Table I. Demographic characteristics and clinical data (values presented as means \pm standard deviation).

\begin{tabular}{lccc}
\hline & \multicolumn{2}{c}{ Group } \\
\cline { 2 - 3 } & $\begin{array}{c}\text { Magnesium } \\
(\mathrm{n}=30)\end{array}$ & $\begin{array}{c}\text { Control } \\
(\mathrm{n}=30)\end{array}$ & P-value \\
Parameter & $66.2 \pm 9.8$ & $65.1 \pm 10.9$ & 0.683 \\
\cline { 2 - 3 } Age (years) & & & 0.299 \\
Gender & 11 & 16 & \\
Male & 19 & 14 & \\
Female & $26.3 \pm 3.0$ & $26.0 \pm 3.2$ & 0.675 \\
Body mass index (kg/m²) & & & 0.067 \\
Diagnosis (n) & 21 & 13 & \\
Osteoarthritis & 9 & 17 & \\
Aseptic necrosis & $76.0 \pm 16.8$ & $74.5 \pm 17.1$ & 0.721 \\
Length of surgery (min) & & & \\
Serum magnesium & & & \\
concentration (mmol/l) & & & \\
Preoperative & $1.00 \pm 0.15$ & $1.03 \pm 0.15$ & 0.376 \\
Postoperative, 6 h & $1.08 \pm 0.17$ & $1.04 \pm 0.15$ & 0.414 \\
Postoperative, 24 h & $1.02 \pm 0.18$ & $1.04 \pm 0.17$ & 0.632 \\
Total drainage fluid (ml) & $428.3 \pm 81.2$ & $409.0 \pm 82.4$ & 0.364 \\
\hline
\end{tabular}

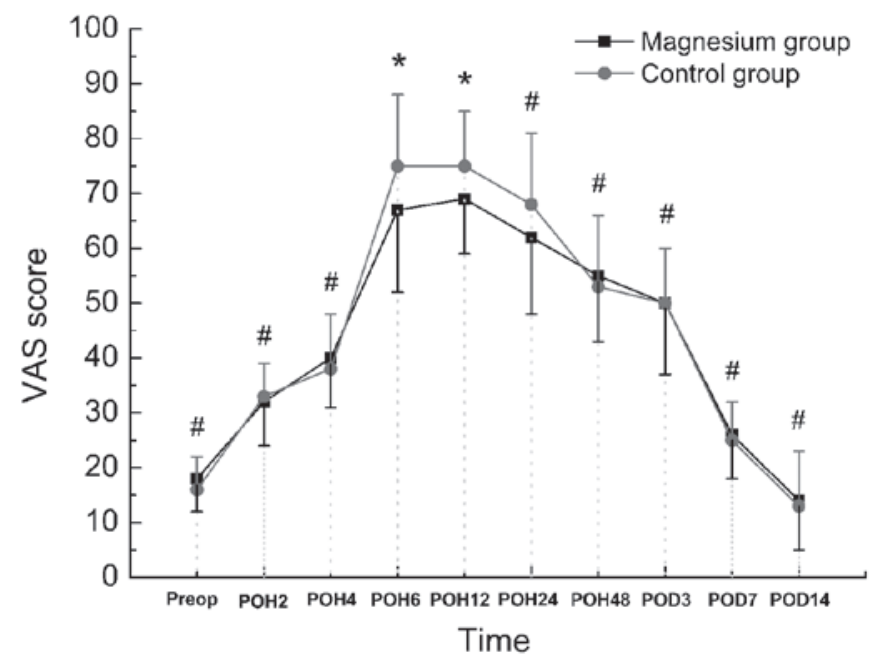

Figure 1. VAS score at rest up to postoperative day $14 .{ }^{*} \mathrm{P}<0.05$ and ${ }^{\#} \mathrm{P}>0.05$. VAS, visual analogue score; $\mathrm{PO}$, postoperative; $\mathrm{H}$, hour; $\mathrm{D}$, day.

hour 6 and 12 in the magnesium group as compared with the control group $(\mathrm{P}<0.05)$, although the difference was insignificant preoperatively and at postoperative hours 2 , 4, 24 and 48, and days 3, 7 and 14 (P>0.05; Fig. 1). This indicator during activity was also lower in the magnesium group at postoperative hour 24 compared with the control group $(\mathrm{P}<0.05)$, although the difference was insignificant preoperatively and at hour 48, and days 7 and 14 ( $\mathrm{P}>0.05$; Fig. 2). Following surgery, the consumption of morphine at $0-6$, 6-12 and 0-48 h (the total quantity) in the magnesium group was significantly lower when compared with the control group $(\mathrm{P}<0.05)$; however, no significant differences were observed

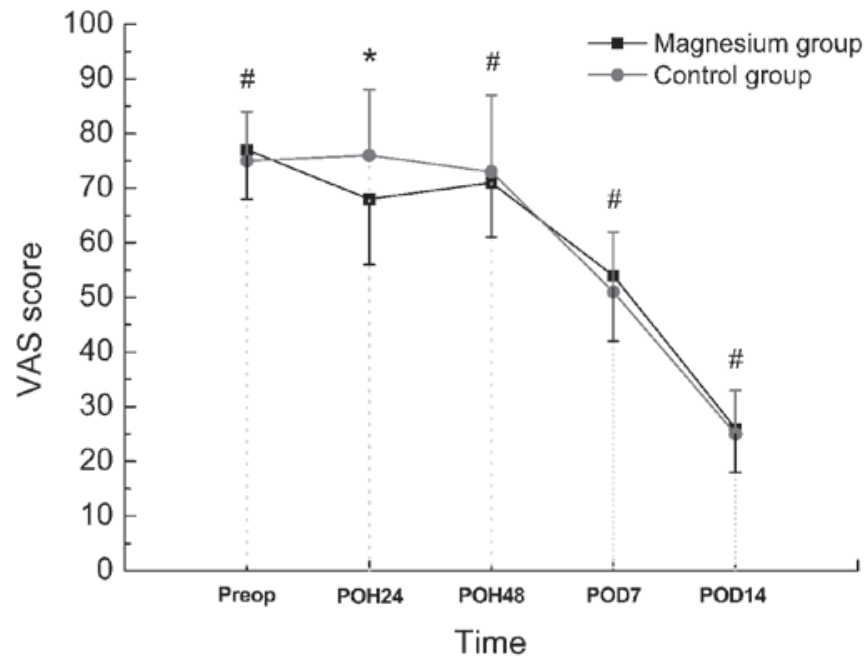

Figure 2. VAS score during activity up to postoperative day 14 . ${ }^{*} \mathrm{P}<0.05$ and ${ }^{\#} \mathrm{P}>0.05$. VAS, visual analogue score; PO, postoperative; H, hour; D, day.

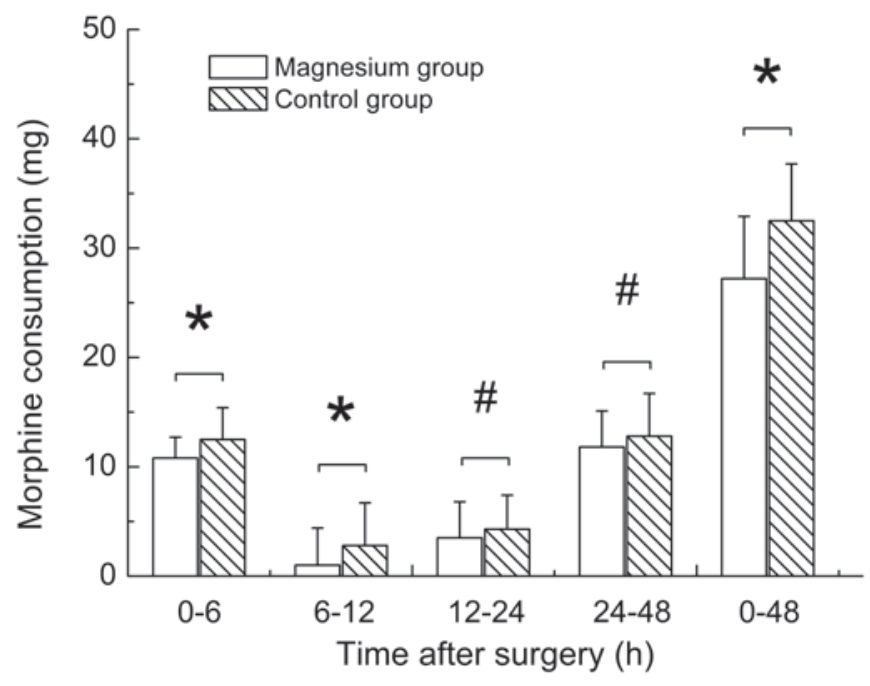

Figure 3. Consumption of morphine up to 48 h post-surgery. ${ }^{*} \mathrm{P}<0.05$ and ${ }^{\#} \mathrm{P}>0.05$.

at 12-24 and 24-48 h between the groups ( $\mathrm{P}>0.05$; Fig. 3 ). The HHS was performed to evaluate postoperative recovery conditions. As shown in Table II, although the improvements from preoperative to postoperative scores were statistically significant $(\mathrm{P}<0.05)$, no significant differences were identified between the two groups $(\mathrm{P}>0.05)$. At the final follow-up, 25 cases in the magnesium group and 26 in the control group achieved excellent or good scores.

AEs. A total of 17 patients (28\%) reported at least one AE and the proportions did not differ according to the treatment group (Table III). Lower extremity Doppler ultrasound scans were performed for every patient three days post-surgery. Two cases in the magnesium group and one in the control group were diagnosed with a DVT of the lower extremity. These patients remained under observation for two weeks and no serious AEs, such as pulmonary embolism or limb amputation occurred. Those patients were advised to attend periodic outpatient follow-up. 
Table II. Outcome of Harris hip score (values presented as means \pm standard deviation).

\begin{tabular}{|c|c|c|c|c|c|c|}
\hline \multirow[b]{2}{*}{ Time } & \multirow[b]{2}{*}{ Group } & \multicolumn{4}{|c|}{ Rating grade } & \multirow[b]{2}{*}{ P-value } \\
\hline & & Excellent (90-100) & Good (80-89) & Fair (70-79) & Poor $(<70)$ & \\
\hline \multirow[t]{2}{*}{ Preoperative } & Magnesium & 0 & 0 & 11 & 19 & \multirow[t]{2}{*}{0.491} \\
\hline & Control & 0 & 1 & 13 & 16 & \\
\hline \multirow[t]{2}{*}{ Postoperative day 7} & Magnesium & 4 & 15 & 11 & 0 & \multirow[t]{2}{*}{0.279} \\
\hline & Control & 2 & 21 & 7 & 0 & \\
\hline \multirow[t]{2}{*}{ Postoperative day 14} & Magnesium & 7 & 18 & 5 & 0 & \multirow[t]{2}{*}{0.823} \\
\hline & Control & 9 & 17 & 4 & 0 & \\
\hline
\end{tabular}

Table III. Adverse events.

\begin{tabular}{llll}
\hline \multirow{2}{*}{ Adverse event } & \multicolumn{2}{c}{ Group (n=30/group) } & \\
\cline { 2 - 3 } & $\begin{array}{c}\text { Magnesium, } \\
\mathrm{n}(\%)\end{array}$ & $\begin{array}{c}\text { Control, } \\
\mathrm{n}(\%)\end{array}$ & P-value \\
\hline Deep venous thrombosis & $2(6.7)$ & $1(3.3)$ & 1.000 \\
Vomiting & $1(3.3)$ & $0(0.0)$ & 1.000 \\
Nausea & $5(16.7)$ & $3(10.0)$ & 0.706 \\
Dizziness & $4(13.3)$ & $6(20.0)$ & 0.731 \\
Headache & $2(6.7)$ & $2(6.7)$ & 1.000 \\
Urine retention & $0(0.0)$ & $1(3.3)$ & 1.000 \\
\hline
\end{tabular}

\section{Discussion}

The present study indicates that intra-articular injection of magnesium sulphate exerted analgesic effects following THA, which decreased postoperative pain and morphine consumption in the early stage of recovery either at rest or during activity.

In recent years, as a result of increasing numbers of osteoarthritis cases and other degenerative disorders of the joint, THA is being widely performed and has been demonstrated to be a highly successful surgical intervention $(16,17)$. Although this procedure is effective, it is often associated with a delayed rehabilitation and prolonged hospitalization, and is accompanied by severe pain during the early postoperative period. At present, various medications are available for postoperative pain control in orthopedic surgeries, among which magnesium is ideal, as it is safe and inexpensive. Although the underlying mechanism of the antinociceptive effect remains unclear, its interference with NMDA receptors and calcium channels appears to affect postoperative pain. Previous studies revealed that NMDA receptors exerted excitatory synaptic transmission effects and possessed negative modulatory sites, and could be blocked by magnesium in a voltage-dependent manner $(18,19)$. In animal models, NMDA receptor antagonists have been shown to have analgesic properties in pain conditions, with similar effects also observed in humans $(20,21)$. Conversely, calcium has been shown to be associated with the release of neurotransmitters and substances implicated in nociceptive response (22), thus the blockage of calcium channels may initiate the pain relief process (23).
Magnesium has been shown to exert its analgesic effect by peripheral, intravenous or spinal infusion (24). With the advantages of simplicity and minimal risk for complications, intra-articular administration of magnesium has received increasing attention and interest. There is evidence that NMDA-receptor exists in the peripheral terminal of articular primary afferent fibers and cellular elements of the joint (25). Previous studies revealed that magnesium exerted its possible anti-nociceptive effect predominantly via peripheral NMDA-receptor mechanisms when administrated locally (26-28). Therefore, the method of intra-articular injection was adopted in the present study.

The analgesic effect of magnesium by intra-articular injection has been confirmed by various studies. Koltka et al (29) conducted a randomized clinical study on 120 patients undergoing arthroscopic meniscectomy. In their study, the patients received magnesium sulphate $(500 \mathrm{mg}$ diluted in $20 \mathrm{ml}$ normal saline), levobupivacaine ( $100 \mathrm{mg}$ diluted in $20 \mathrm{ml}$ normal saline), lornoxicam (8 mg diluted in $20 \mathrm{ml}$ normal saline) or $20 \mathrm{ml}$ normal saline by intra-articular injection prior to tourniquet deflation. Koltka et al (29) found that magnesium sulphate was a more effective analgesic than the placebo, although the most effective was lornoxicam (29). In another study performed by Bondok and Abd El-Hady (12), a total of 60 cases undergoing arthroscopic knee surgery were randomly allocated to receive intra-articular injection of either $10 \mathrm{ml}$ magnesium sulphate $(50 \mathrm{mg} / \mathrm{ml})$ or $10 \mathrm{ml}$ normal saline prior to tourniquet release. It was observed that the magnesium group obtained a significant reduction in pain scores and less total diclofenac consumption when compared with the control group postoperatively. It was concluded that intra-articular magnesium administration was effective for postoperative analgesia (12). In the present study, intra-articular administration of magnesium was performed for pain management following THA, and the results indicated that this strategy improved postoperative pain scores when compared with the control group, which was consistent with the above-mentioned reports.

No significant differences in preoperative data, surgical duration and total drainage fluid were identified between the two groups, nor were there differences in intraoperative anesthesia, surgical approach and the types of implant; therefore, associated factors that may affect postoperative VAS and rehabilitation were excluded. The results of VAS at rest, at postoperative hours 2 and 4 did not differ depending on the treatment type, and the two were smaller than that at hour 6 , 
which may be due to residual anesthetic in the circulatory system. No significant differences in VAS were found after postoperative day 2, as magnesium would be absorbed and metabolized by the body. The result of HHS indicated that intra-articular administration of magnesium may not facilitate functional recovery in the early stages of recovery. However, the outcomes of VAS at postoperative hours 6 and 12 at rest, and postoperative hour 24 during activity were significantly lower in the magnesium group, and the consumption of morphine at $0-6,6-12$ and $0-48 \mathrm{~h}$ were also significantly reduced. Thus, it was concluded that magnesium reduces postoperative pain when administered intra-articularly following THA as compared with administration of normal saline.

In the present study, serum magnesium concentrations were not influenced by intra-articular injection at a low dose $(1 \mathrm{~g})$, as the levels were comparable with those of the control group, postoperatively and following surgery. All the serum magnesium concentrations were within normal limits and no associated AE was observed.

There were certain limitations of the present study. A drainage system was applied and a small quantity of magnesium sulphate was able to exude from it, thus it was hypothesized that a lack of drainage could have contributed to greater analgesia efficacy. In addition, the present study was monocentric; therefore, the results may be biased, and a multicenter study may strengthen the generalizability of the outcomes. Furthermore, the present study was limited to the early postoperative analgesia and complications, thus further studies of subsequent clinical outcomes are required.

In conclusion, the present study indicates that the administration of intra-articular magnesium sulphate provides improved pain control and reduces the requirement for morphine in the early postoperative period without increasing short-term complications, when compared with a placebo.

\section{References}

1. McKenzie JC, Goyal N and Hozack WJ: Multimodal pain management for total hip arthroplasty. Seminars in Arthroplasty 24: 87-93, 2013

2. Young AC and Buvanendran A: Pain management for total hip arthroplasty. J Surg Orthop Adv 23: 13-21, 2014.

3. Badica CIG, Badica L, Grecu I, Bradis AA and Grintescu IM: Opioids or NSAID in postoperative analgesia after total hip arthroplasty? Comparison of adverse events: 14AP4-4. Eur J Anaesthesiol 24: 177-178, 2007.

4. Fransen M and Neal B: Non-steroidal anti-inflammatory drugs for preventing heterotopic bone formation after hip arthroplasty. Cochrane Database Syst Rev 2004: CD001160, 2004.

5. Kuchálik J, Granath B, Ljunggren A, Magnuson A, Lundin A and Gupta A: Postoperative pain relief after total hip arthroplasty: A randomized, double-blind comparison between intrathecal morphine and local infiltration analgesia. Br J Anaesth 111: 793-799, 2013.

6. Soave PM, Conti G, Costa R and Arcangeli A: Magnesium and anaesthesia. Curr Drug Targets 10: 734-743, 2009.

7. Abdulatif M, Amin SMM, Aboul-Ela A, Samuel EWM and Abdel-Hakim SMA: Intra-articular versus intravenous magnesium-sulfate as adjuvant to femoral nerve block in arthroscopic knee surgery under general anesthesia: Randomized controlled trial. Egypt J Anaesth 31: 239-246, 2015.

8. Saritas TB, Borazan H, Okesli S, Yel M and Otelcioglu Ş: Is intra-articular magnesium effective for postoperative analgesia in arthroscopic shoulder surgery? Pain Res Manag 20: 35-38, 2015 .
9. Ekmekci P, Bengisun ZK, Akan B, Kazbek BK, Ozkan KS and Suer AH: The effect of magnesium added to levobupivacaine for femoral nerve block on postoperative analgesia in patients undergoing ACL reconstruction. Knee Surg Sports Traumatol Arthrosc 21: 1119-1124, 2013.

10. Farouk S and Aly A: A comparison of intra-articular magnesium and/or morphine with bupivacaine for postoperative analgesia after arthroscopic knee surgery. J Anesth 23: 508-512, 2009.

11. Lee AR, Yi HW, Chung IS, Ko JS, Ahn HJ, Gwak MS, Choi DH and Choi SJ: Magnesium added to bupivacaine prolongs the duration of analgesia after interscalene nerve block. Can J Anaesth 59: 21-27, 2012.

12. Bondok RS and Abd El-Hady AM: Intra-articular magnesium is effective for postoperative analgesia in arthroscopic knee surgery. Br J Anaesth 97: 389-392, 2006.

13. Elsharnouby NM, Eid HE, Abou Elezz NF and Moharram AN: Intraarticular injection of magnesium sulphate and/or bupivacaine for postoperative analgesia after arthroscopic knee surgery. Anesth Analg 106: 1548-1552, 2008.

14. Rozner MA: The American Society of Anesthesiologists physical status score and risk of perioperative infection. JAMA 275: 1544-1544, 1996.

15. Mahomed NN, Arndt DC, McGrory BJ and Harris WH: The Harris hip score: Comparison of patient self-report with surgeon assessment. J Arthroplasty 16: 575-580, 2001.

16. Kim SJ, Huh J, Odrobina R and Kim JH: Systemic review of published literature on Candida infection following total hip arthroplasty. Mycopathologia 179: 173-185, 2015.

17. Tsertsvadze A, Grove A, Freeman K, Court R, Johnson S, Connock M, Clarke A and Sutcliffe P: Total hip replacement for the treatment of end stage arthritis of the hip: A systematic review and meta-analysis. PLoS One 9: e99804, 2014.

18. Ascher P and Nowak L: Electrophysiological studies of NMDA receptors. Trends Neurosci 10: 284-288, 1987.

19. Coderre TJ, Katz J, Vaccarino AL and Melzack R: Contribution of central neuroplasticity to pathological pain: Review of clinical and experimental evidence. Pain 52: 259-285, 1993.

20. Fisher K, Coderre TJ and Hagen NA: Targeting the $\mathrm{N}$-methyl-D-aspartate receptor for chronic pain management. Preclinical animal studies, recent clinical experience and future research directions. J Pain Symptom Manage 20: 358-373, 2000.

21. Hewitt DJ: The use of NMDA-receptor antagonists in the treatment of chronic pain. Clin J Pain 16 (Suppl 2): S73-S79, 2000.

22. Memiş D, Turan A, Karamanlioğlu B, Süt N and Pamukçu Z: The use of magnesium sulfate to prevent pain on injection of propofol. Anesth Analg 95: 606-608, 2002.

23. Silverman R, Bendick PJ and Wasvary HJ: A randomized, prospective, double-blind, placebo-controlled trial of the effect of a calcium channel blocker ointment on pain after hemorrhoidectomy. Dis Colon Rectum 48: 1913-1916, 2005.

24. Arcioni R, Palmisani S, Tigano S, Santorsola C, Sauli V, Romanò S, Mercieri M, Masciangelo R, De Blasi RA and Pinto G: Combined intrathecal and epidural magnesium sulfate supplementation of spinal anesthesia to reduce post-operative analgesic requirements: A prospective, randomized, double-blind, controlled trial in patients undergoing major orthopedic surgery. Acta Anaesthesiol Scand 51: 482-489, 2007.

25. Lawand NB, Willis WD and Westlund KN: Excitatory amino acid receptor involvement in peripheral nociceptive transmission in rats. Eur J Pharmacol 324: 169-177, 1997.

26. Mercieri M, De Blasi RA, Palmisani S, Forte S, Cardelli P, Romano R, Pinto G and Arcioni R: Changes in cerebrospinal fluid magnesium levels in patients undergoing spinal anaesthesia for hip arthroplasty: Does intravenous infusion of magnesium sulphate make any difference? A prospective, randomized, controlled study. Br J Anaesth 109: 208-215, 2012.

27. Samir EM, Badawy SS and Hassan AR: Intrathecal vs intravenous magnesium as an adjuvant to bupivacaine spinal anesthesia for total hip arthroplasty. Egypt J Anaesth 29: 395-400, 2013.

28. Lin CY, Tsai PS, Hung YC and Huang CJ: L-type calcium channels are involved in mediating the anti-inflammatory effects of magnesium sulphate. Br J Anaesth 104: 44-51, 2010.

29. Koltka K, Koknel-Talu G, Asik M and Ozyalcin S: Comparison of efficacy of intraarticular application of magnesium, levobupivacaine and lornoxicam with placebo in arthroscopic surgery. Knee Surg Sports Traumatol Arthrosc 19: 1884-1889, 2011. 